\title{
Manejo terapêutico na deficiência de cálcio: uma revisão sistemática
}

\author{
Ana Paula Anzolin*, Silvia Cristina Fagundes ${ }^{\star *}$, Micheila Alana Fagundes ${ }^{\star \star}$, Gabriela Dal Pizzol ${ }^{\star \star *}$, \\ Marcos Roberto Spassim*, Charise Dallazem Bertol ${ }^{*, * *}$
}

\section{Resumo}

A perda de massa óssea gradual origina osteopenia e sua evolução leva a osteoporose. Ocorre diminuição da massa óssea e deterioração da microarquitetura do tecido ósseo, aumento da fragilidade óssea e da susceptibilidade a fraturas. O objetivo deste artigo foi avaliar as diferenças entre as apresentações de cálcio disponíveis comercialmente quanto a sua eficácia, segurança e custos. Foi realizada uma revisão sistemática, utilizando as palavras-chave: "calcium", "osteoporosis", "doses", "treatment", "randomised", "controlled" e "meta-analysis". A seleção das publicações foi feita a partir de critérios de inclusão e exclusão. Ao total 379 artigos relevantes foram identificados. Destes, 10 preencheram os critérios de seleção e foram incluídos neste estudo. Entre os sais de cálcio, encontra-se o citrato, carbonato, fluoreto, folinato, fosfato, e de ostras, e também são usados os bifosfonados. Cálcio associado a vitamina $\mathrm{D}$ e magnésio também é usado. $\mathrm{O}$ uso do cálcio apresenta muitos benefícios na prevenção e tratamento da osteoporose, especialmente ao ser associado ao magnésio e vitamina D. O citrato de cálcio mostrou-se como a melhor opção de compra, visto que pode ser usado por idosos com problemas renais e também por pacientes que utilizam antiácidos, mostrando uma absorção e biodisponibilidade superior ao carbonato, sendo a escolha com o menor preço encontrado.

Palavras Chaves: Cálcio; Osteoporose; Tratamento; Farmacoeconomia; Idosos.

\section{Introdução}

A perda de massa óssea gradual origina osteopenia (baixa densidade óssea) que, que tem como consequência grave a osteoporose. A osteopenia nem sempre está associada a doença pois existem pessoas que naturalmente têm baixa densidade óssea (COMPSTON et al., 2003).

* Programa de Pós-Graduação em Envelhecimento Humano. Universidade de Passo Fundo. Rio Grande do Sul. Brasil.

** Curso de Farmácia. Universidade de Passo Fundo. Rio Grande do Sul. Brasil.

**** Curso de Pós-Graduação em Bioexperimentação. Universidade de Passo Fundo. Rio Grande do Sul. Brasil.

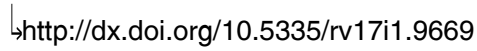


Na osteoporose há diminuição da massa óssea, deterioração da microarquitetura do tecido ósseo com aumento da fragilidade óssea e da susceptibilidade a fraturas (KANIS, 2002). É uma doença osteometabólica, em que a densidade mineral óssea (DMO) é igual ou inferior a 2,5 desvios padrão abaixo do pico de massa óssea encontrada no adulto jovem, e a osteopenia apresenta DMO entre 1 a 2,5 desvios padrão abaixo do pico de massa óssea encontrada no adulto jovem (BORISSOVA, 2013).

A definição clínica da doença se baseia tanto na evidência de fratura como na medida da DMO. Além de fraturas, os sintomas incluem dor, depressão, deformidade, perda da independência e aumento da mortalidade (KANIS et al., 2008).

A osteoporose é classificada em: primária e a secundária. A primária ocorre com o avançar da idade como consequência do efeito acumulativo da perda e deterioração da massa óssea, e afeta essencialmente os idosos. A secundária define-se como uma alteração da microarquitetura e perda da massa óssea em decorrência de uma doença subjacente ou uso abusivo de glicocorticoides (GALI, 2001).

Dados epidemiológicos mostram que $50 \%$ das mulheres e $20 \%$ dos homens com idade superior ou igual a 50 anos sofrerão uma fratura decorrente da osteoporose. No Brasil, em 1980 cerca de 7,5 milhões de pessoas tinham a doença e em 2000 já eram 15 milhões (CARVALHO; FONSECA; PEDROSA, 2004). Há muitas mortes decorrentes de fraturas, como por exemplo: $20 \%$ dos idosos morrem um ano após ter uma fratura de quadril (KANIS, 2008).

As principais células que compõem o tecido ósseo são os osteoblastos, osteócitos e osteoclastos, responsáveis pela criação, manutenção, secreção de substâncias para a manutenção dos ossos na matriz extracelular, e reabsorção do tecido ósseo. Portanto precisam estar em constante metabolismo, pois qualquer desequilíbrio ocasiona alteração do tecido ósseo, como é o caso na pós-menopausa em que a atividade osteoclástica é mais intensa e há grande probabilidade de desenvolver osteoporose (CAETANO-LOPES; CANHÃO; FONSECA, 2007).

A alteração hormonal também é discutida na osteoporose. $\mathrm{O}$ excesso de atividade do hormônio da paratiróide (paratormônio-PTH), diagnosticado como hiperparatiroidismo, é uma das causas de osteoporose (GRACITELLI et al., 2002).

Algumas terapias buscam reparar ou prevenir o déficit nutricional da massa óssea. Um dos componentes dietéticos de grande importância para o tecido ósseo é o cálcio.

Com o envelhecimento populacional e a maior probabilidade de mais indivíduos desenvolverem osteoporose, medidas de intervenção e tratamento devem ser adotados, prevenindo perdas ósseas e risco de fraturas.

A presente revisão tem como objetivo avaliar as diferenças entre as apresentações de cálcio disponíveis no mercado 
quanto a sua eficácia, segurança e custos.

\section{Metodologia}

A pesquisa foi realizada nos bancos de dados Medline via Pubmed, Cochrane Controlled Trial Register e Cochrane Databases Systematic Reviews (Cochrane Library), no período de 1990 a 2018, utilizando as palavras-chaves calcium, osteoporosis, doses, treatment, randomised, controlled e meta-analysis.

Foram incluídos todos os estudos disponíveis, na íntegra, que informaram:

- Ensaios Clínicos randomizados onde o objetivo principal era o tratamento da osteoporose.

- Revisões sistemáticas ou meta-análises de ensaios clínicos controlados randomizados ou pré-clínicos que avaliam o uso de cálcio e suas combinações na osteoporose (ou osteoporose induzida).

- Estudos que apresentam como desfecho primário a avaliação do uso do cálcio e suas reações adversas.

- Artigos disponíveis na língua portuguesa, inglesa ou espanhola.

Os critérios de exclusão foram estudos que avaliaram o uso do cálcio com outro enfoque que não a osteoporose. Textos não disponíveis na íntegra.

\section{Resultados}

Na primeira busca foram identificados 9.800 artigos. Após critérios como ano entre 1990 a 2018, tipo de estudo, língua e disponibilidade na íntegra foram encontrados 379 artigos relevantes. Após a revisão do título e resumo, e com duplicações descartadas, o total foi reduzido para 78 documentos potencialmente relevantes. Destes, após triagem de texto completo foram selecionados 10 artigos dos quais preencheram os critérios de seleção e foram incluídos na elaboração da revisão sistemática. A Figura 1 mostra um resumo do fluxo de pesquisa na literatura e na seleção de estudos utilizados. 
Figura 1: Pesquisa de literatura e fluxo de seleção

\section{Identificação}

MEDLINE, Cochrane Controlled Trial Register e Cochrane Databases Systematic Reviews (Cochrane Library)

$$
(n=9.800)
$$

Busca: (((CALCIUM) AND OSTEOPOROSIS) AND TREATMENT)

\section{Seleção \\ 1a seleção: Estudos disponíveis na integra, ensaios clínicos, ensaio clínico controlado e meta-análises. Línguas espanhol, inglês e português. Texto no período de 1990 a 2018.}

( $n=379)$

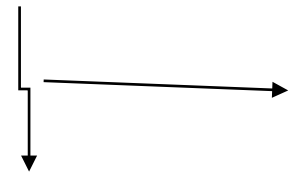

Estudos Excluídos, após ler título e resumo e não preencherem critérios de inclusão ( $n=301)$

\section{Elegibilidade}

\section{Incluídos}

Estudos Selecionados para leitura na íntegra $(n=78)$

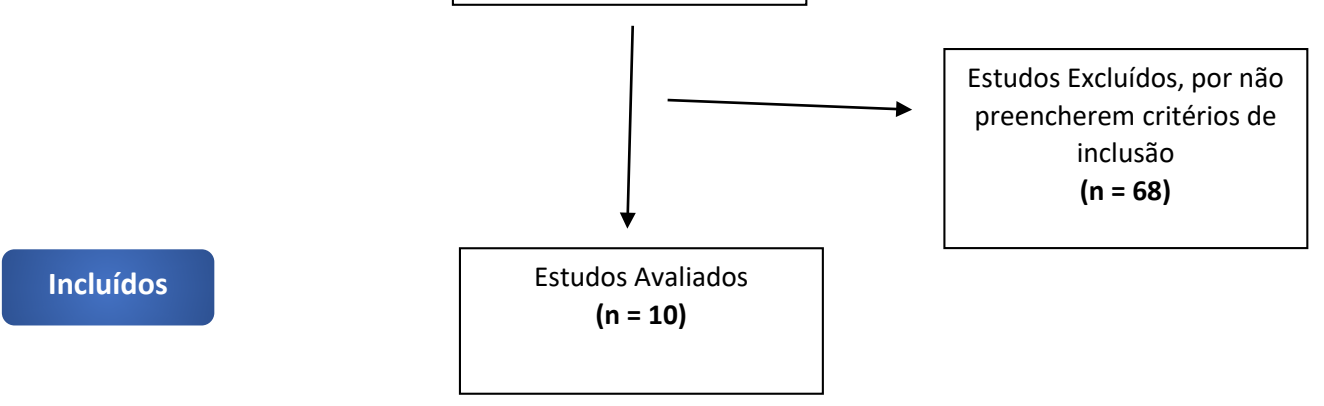

Fonte: autor (2019)

Entre os sais de cálcio, encontra-se o citrato, carbonato, magnésio, alendronato, de ostras, fluoreto, folinato e fosfato. Esses sais apresentam diferenças quanto suas características químicas, farmacocinéticas e farmacodinâmicas.
No quadro 1, encontram-se os ensaios clínicos e pré-clínicos onde foi comparado os resultados de eficácia dos diferentes sais de cálcio e no quadro 2 foi explanada a diferença entre os diferentes sais de cálcio, considerando o cálcio elementar, modo de utilização e o preço máximo ao consumidor (PMC). 
Quadro 1: Resumo dos estudos avaliados relacionados ao cálcio e suas diferenças na metodologia e conclusões

\begin{tabular}{|c|c|c|c|}
\hline Referência & Objetivo & Delineamento do estudo & Resultados e Conclusão \\
\hline $\begin{array}{l}\text { (HARVEY; } \\
\text { ZOBITZ; } \\
\text { PAK, 1988) }\end{array}$ & $\begin{array}{l}\text { Comparar citrato } \\
\text { com carbonato } \\
\text { de cálcio em je- } \\
\text { jum }\end{array}$ & $\begin{array}{l}\text { Estudo duplo-cego, com } 20 \text { mulheres. A absorção } \\
\text { do cálcio foi medida pela técnica de contagem ex- } \\
\text { terna do antebraço, onde a relação entre a radio- } \\
\text { atividade do antebraço após uma dose oral de sal } \\
\text { de cálcio marcado (contendo } 500 \text { mg de cálcio) } \\
\text { e após uma injeção intravenosa de radiocalcium } \\
\text { representou absorção fracionada de cálcio. }\end{array}$ & $\begin{array}{l}\text { O citrato de cálcio apresenta absorção superior ao carbo- } \\
\text { nato de cálcio, quando ingerido com o estômago vazio. A } \\
\text { absorção do citrato de cálcio foi de } 39,2 \% \text {, significativamen- } \\
\text { te maior que o carbonato de cálcio que mostrou absorção } \\
\text { de } 31,2 \% \text {. }\end{array}$ \\
\hline $\begin{array}{l}\text { (KENNY et } \\
\text { al., 2004) }\end{array}$ & $\begin{array}{l}\text { Comparar citrato } \\
\text { com o carbona- } \\
\text { to de cálcio nos } \\
\text { marcadores de } \\
\text { reabsorção ós- } \\
\text { sea em mulheres } \\
\text { pós-menopausa. }\end{array}$ & $\begin{array}{l}\text { Estudo cruzado, randomizado, aberto, com } 40 \\
\text { mulheres. Receberam } 1000 \mathrm{mg} / \text { dia de citrato de } \\
\text { cálcio ou carbonato de cálcio por } 12 \text { semanas, se- } \\
\text { guidas por } 2 \text { semanas sem suplementos de cálcio } \\
\text { e } 12 \text { semanas de tratamento com o suplemento } \\
\text { alternativo de cálcio. Todas as mulheres recebe- } \\
\text { ram vitamina D ( } 900 \text { Ul / dia). }\end{array}$ & $\begin{array}{l}\text { O citrato de cálcio diminuiu os marcadores de reabsorção } \\
\text { óssea significativamente mais do que o carbonato de cálcio } \\
\text { em mulheres na pós-menopausa, embora não tenham sido } \\
\text { detectadas diferenças em seus efeitos na excreção de cál- } \\
\text { cio ou no PTH. }\end{array}$ \\
\hline
\end{tabular}


(continuação...)

\begin{tabular}{|c|c|c|c|}
\hline $\begin{array}{l}\text { (HOWARD et } \\
\text { al., 2000) }\end{array}$ & $\begin{array}{l}\text { Comparar a bio- } \\
\text { disponibilidade } \\
\text { de dose única e } \\
\text { os efeitos sobre } \\
\text { a função da pa- } \\
\text { ratireoide de dois } \\
\text { suplementos de } \\
\text { citrato de cálcio + } \\
\text { vitamina D e car- } \\
\text { bonato de cálcio. }\end{array}$ & $\begin{array}{l}\text { Estudo randomizado com } 25 \text { mulheres pós-meno- } \\
\text { páusicas Efeitos de uma única dose de carbonato } \\
\text { de cálcio, citrato de cálcio + vitamina D, ou pla- } \\
\text { cebo. } \\
\text { Amostras de sangue foram coletadas de hora em } \\
\text { hora por } 4 \text { ou } 6 \text { horas após cada dose. }\end{array}$ & $\begin{array}{l}\text { O citrato de cálcio forneceu uma variação pico-basal } 46 \% \\
\text { maior e uma mudança } 94 \% \text { maior na área sob a curva } \\
\text { (ASC) para o cálcio sérico e um incremento de } 41 \% \text { no cál- } \\
\text { cio urinário em relação ao carbonato de cálcio. } \\
\text { O decréscimo da concentração do PTH em relação à quan- } \\
\text { tidade inicial foi maior após o uso do citrato de cálcio. } \\
\text { A ASC do cálcio no sangue foi maior após o uso de citrato } \\
\text { do que o do carbonato, demonstrando maior biodisponibili- } \\
\text { dade do citrato em relação ao carbonato, quando adminis- } \\
\text { trado com uma refeição. }\end{array}$ \\
\hline $\begin{array}{l}\text { (HELLER et } \\
\text { al, 1999) }\end{array}$ & $\begin{array}{l}\text { Comparar os ín- } \\
\text { dices farmacoci- } \\
\text { néticos de absor- } \\
\text { ção de citrato de } \\
\text { cálcio + vitamina } \\
\text { D e carbonato de } \\
\text { cálcio. }\end{array}$ & $\begin{array}{l}\text { Ensaio clínico com } 18 \text { mulheres pós-menopausa } \\
\text { divididas em três grupos, que receberam uma } \\
\text { dose de } 500 \text { mg de citrato de cálcio ou carbonato } \\
\text { de cálcio ou placebo. } \\
\text { A concentração sanguínea do cálcio foi monitora- } \\
\text { da antes da administração, e, de hora em hora por } \\
6 \text { horas pós-administração. }\end{array}$ & $\begin{array}{l}\text { A ASC do cálcio sérico foi } 2,5 \text { vezes maior para citrato de } \\
\text { cálcio do que para carbonato, e a variação na concentração } \\
\text { do cálcio sérico em relação à concentração antes da admi- } \\
\text { nistração foi } 76 \% \text { maior para o citrato. } \\
\text { Em todos os tempos após a administração o citrato de cál- } \\
\text { cio apresentou uma concentração de cálcio significativa- } \\
\text { mente maior do que o carbonato. } \\
\text { A ASC e a concentração de carbonato de cálcio encontrada } \\
\text { após a administração não foi significativamente diferente } \\
\text { do placebo, e, o incremento no cálcio sérico somente fo } \\
\text { significativo em relação ao placebo após } 6 \text { horas da admi- } \\
\text { nistração. } \\
\text { Portanto, foi comprovado que o citrato de cálcio é mais bio- } \\
\text { disponível que o carbonato de cálcio. }\end{array}$ \\
\hline
\end{tabular}


(continuação...)

\begin{tabular}{|c|c|c|c|}
\hline $\begin{array}{l}\text { (KRÖGER, et } \\
\text { al., 2010) }\end{array}$ & $\begin{array}{l}\text { Verificar se a su- } \\
\text { plementação de } \\
\text { vitamina D e cál- } \\
\text { cio pode prevenir } \\
\text { a perda óssea } \\
\text { em mulheres na } \\
\text { pós-menopausa. }\end{array}$ & $\begin{array}{l}\text { Ensaio clínico aberto randomizado, de } 593 \text { mulhe- } \\
\text { res idosas. } \\
\text { O grupo suplementação }(n=287) \text { recebeu diaria- } \\
\text { mente colecalciferol } 800 \text { Ul + carbonado de cálcio } \\
1.000 \mathrm{mg} \text { por } 3 \text { anos, enquanto o grupo controle } \\
(n=306) \text { não recebeu suplementação nem pla- } \\
\text { cebo. } \\
\text { Todas foram submetidas a densitometria óssea. }\end{array}$ & $\begin{array}{l}\text { A suplementação diária de vitamina } D \text { e cálcio tem um efeito } \\
\text { positivo sobre o esqueleto em mulheres ambulatoriais na } \\
\text { pós-menopausa com ingestão nutricional de cálcio adequa- } \\
\text { da. } \\
\text { Mulheres aderentes ( } \geq 80 \% \text { de uso) tiveram efeitos melho- } \\
\text { res e estatisticamente significativos nas regiões total do cor- } \\
\text { po e femoral. }\end{array}$ \\
\hline $\begin{array}{l}\text { (SERVICES } \\
\text { et al., } \\
\text { 2012) }\end{array}$ & $\begin{array}{l}\text { Avaliar os efei- } \\
\text { tos do cálcio na } \\
\text { densidade óssea } \\
\text { e fraturas em } \\
\text { mulheres na pós- } \\
\text {-menopausa. }\end{array}$ & $\begin{array}{l}\text { Realização de uma meta-análise, onde foram } \\
\text { incluídos } 15 \text { ensaios clínicos num total de } 1806 \\
\text { pacientes. }\end{array}$ & $\begin{array}{l}\text { Em } 2 \text { anos de tratamento com cálcio elementar, houve dis- } \\
\text { creta proteção no risco de fraturas vertebrais em relação ao } \\
\text { grupo placebo, entretanto não ficou claro se houve redução } \\
\text { na incidência de fraturas não-vertebrais. } \\
\text { O carbonato de cálcio apresentou um efeito maior, em evi- } \\
\text { tar fraturas vertebrais, que o citrato de cálcio no osso total } \\
\text { do corpo e no quadril, e, na coluna lombar o efeito foi maior } \\
\text { com o citrato de cálcio. }\end{array}$ \\
\hline
\end{tabular}


(conclusão)

\begin{tabular}{|c|c|c|c|}
\hline $\begin{array}{l}\text { (AGRIPINO, } \\
\text { 2016) }\end{array}$ & $\begin{array}{l}\text { Avaliar a eficácia } \\
\text { e a segurança de } \\
\text { um suplemento } \\
\text { de pó de concha } \\
\text { de ostra (carbo- } \\
\text { nato de cálcio) e } \\
\text { de goma guar (fi- } \\
\text { bra hidrossolúvel) } \\
\text { numa baixa dose. }\end{array}$ & $\begin{array}{l}\text { Foram utilizados } 42 \text { ratos Wistar fêmeas, normo e } \\
\text { hiperglicêmicos. Foram divididos em } 6 \text { grupos (1: } \\
\text { controle; 2:ovariectomizados; } 3 \text { : } \\
\text { Hiperglicêmicos; 4: hiperglicémicos ovariectomi- } \\
\text { zados; } 5 \text { : Ovariectomizados } \\
\text { suplementados e } 6: \text { hiperglicémicos ovariectomi- } \\
\text { zados suplementados). } \\
\text { A dose equivalente em humanos foi de } 420 \mathrm{mg} / \text { dia } \\
\text { de cálcio e } 414 \text { mg/dia de goma guaraná, durante } \\
53 \text { dias. }\end{array}$ & $\begin{array}{l}\text { O cálcio de ostra, mesmo em doses baixas, atenua a calciú- } \\
\text { ria, hipocalcemia, diminuição da espessura do osso cortical } \\
\text { da tíbia e a desmineralização do fémur, tornando-o mais } \\
\text { rígido e resistente sem risco aparente de lesão ou formação } \\
\text { de depósitos vasculares, hepáticos ou renais. } \\
\text { Houve preservação da ultraestrutura do osso cortical e ate- } \\
\text { nuação da diminuição da espessura do osso trabecular nos } \\
\text { animais ovariectomizados com hiperglicemia crônica. }\end{array}$ \\
\hline $\begin{array}{l}\text { (KAHWATI et } \\
\text { al., 2018) }\end{array}$ & $\begin{array}{l}\text { Avaliar as evi- } \\
\text { dências dos be- } \\
\text { nefícios e male- } \\
\text { fícios da vitami- } \\
\text { na D, cálcio ou } \\
\text { suplementação } \\
\text { combinada para } \\
\text { a prevenção pri- } \\
\text { mária de fraturas } \\
\text { em adultos da } \\
\text { comunidade. }\end{array}$ & $\begin{array}{l}\text { Pesquisa realizada nos bancos de dados: } \\
\text { PubMed, EMBASE, Cochrane Library e registros } \\
\text { de testes até 2017; referências; e especialistas. A } \\
\text { vigilância continuou até } 2018 \text {. } \\
\text { Foram selecionados ensaios clínicos randomi- } \\
\text { zados em inglês ou estudos observacionais de } \\
\text { suplementação com vitamina D, cálcio ou ambos } \\
\text { entre populações adultas; foram excluídos es- } \\
\text { tudos de populações institucionalizadas ou com } \\
\text { deficiência de vitamina D, osteoporose ou fratura } \\
\text { anterior. } \\
\text { Para a extração e síntese de dados, houve uma } \\
\text { revisão dupla e independente de títulos / resu- } \\
\text { mos e artigos em texto completo e classificação } \\
\text { da qualidade do estudo usando critérios predefi- } \\
\text { nidos. Meta-análise de efeitos aleatórios usada } \\
\text { quando pelo menos três estudos semelhantes } \\
\text { estavam disponíveis. }\end{array}$ & $\begin{array}{l}\text { Foram selecionados } 11 \text { ensaios clínicos. Comparada ao } \\
\text { placebo, a suplementação com vitamina } D \text { diminuiu a inci- } \\
\text { dência total de fraturas, mas não teve associação significa- } \\
\text { tiva com fratura de quadril. } \\
\text { A suplementação com vitamina } D \text { com cálcio não teve efeito } \\
\text { na incidência total de fraturas ou incidência de fraturas do } \\
\text { quadril. A evidência apenas para o cálcio foi limitada, com } \\
\text { apenas } 2 \text { estudos e resultados muito imprecisos. A suple- } \\
\text { mentação com vitamina } D \text { isolada ou com cálcio não teve } \\
\text { efeito significativo na mortalidade por todas as causas ou } \\
\text { doença cardiovascular incidente. A suplementação com } \\
\text { vitamina D com cálcio foi associada a um aumento da in- } \\
\text { cidência de cálculos renais, mas a suplementação apenas } \\
\text { com cálcio não foi associada a um risco aumentado. } \\
\text { Portanto, a suplementação de vitamina } D \text { isolada ou com } \\
\text { cálcio não foi associada à incidência reduzida de fraturas } \\
\text { em adultos da comunidade sem deficiência conhecida de } \\
\text { vitamina } D \text {, osteoporose ou fratura anterior. A vitamina } D \\
\text { com cálcio foi associada a um aumento na incidência de } \\
\text { cálculos renais. }\end{array}$ \\
\hline
\end{tabular}

Referências: HELLER et al, 1999; HOWARD et al., 2000; HEANEY et al., 2001; KENNY et al., 2004; HARVEY; ZOBITZ; PAK, 1988; KRÖGER, et al., 2010; SERVICES et al., 2012; ZENK; FRESTEDT; AGRIPINO, 2016; KUSKOWSK, 2018; KAHWATI et al., 2018. 
Quadro 2: Diferença entre os diferentes sais de cálcio, considerando o cálcio elementar, modo de utilização e o PMC.

\begin{tabular}{|c|c|c|}
\hline & $\begin{array}{l}\text { Percentual de absorção do cálcio } \\
\text { elementar }\end{array}$ & $\begin{array}{l}\text { Preço máximo ao consumidor (PMC) e } \\
\text { modo de administração }\end{array}$ \\
\hline $\begin{array}{l}\text { Citrato de } \\
\text { cálcio }\end{array}$ & $\begin{array}{l}21 \% \\
\text {-Absorve facilmente (CAMPOS; PI- } \\
\text { NHATI, 2013.) }\end{array}$ & $\begin{array}{l}\text { Uma das apresentações disponível no mer- } \\
\text { cado, tem PMC de cerca de } 38,63 \text { reais para } \\
60 \text { sachês. Ou seja, } 0,64 \text { centavos de reais } \\
\text { por dose. } \\
\text { Deve ser ingerido } 1 \text { sachê de } 12 \text { em } 12 \text { horas } \\
\text { ( } 2 \text { vezes ao dia), a qualquer hora do dia ou } \\
\text { da noite, independentemente das refeições. }\end{array}$ \\
\hline $\begin{array}{l}\text { Carbonato } \\
\text { de cálcio }\end{array}$ & $\begin{array}{l}40 \% \\
\text {-Administração concomitante aos } \\
\text { alimentos melhora sua absorção } \\
\text { (ZENK; FRESTEDT; KUSKOWSKI, } \\
\text { 2018) }\end{array}$ & $\begin{array}{l}\text { PMC para o carbonato de cálcio é cerca de } \\
51,07 \text { reais, para } 60 \text { comprimidos. Ou seja, } \\
0,85 \text { centavos de reais por dose. } \\
\text { Deve ser administrado } 1 \text { comprimido ao dia } \\
\text { durante a refeição. }\end{array}$ \\
\hline $\begin{array}{l}\text { Cálcio de } \\
\text { ostras }\end{array}$ & - 40\% (CAMPOS; PINHATI, 2013) & $\begin{array}{l}\text { Cálcio de Ostras não tem PMC, por ser consi- } \\
\text { derado um suplemento, entretanto seu preço } \\
\text { é de cerca de } 53,75 \text { reais, por } 120 \text { comprimi- } \\
\text { dos. Ou seja, } 0,45 \text { centavos de reais por dose. } \\
\text { Deve-se tomar } 1 \text { comprimido duas vezes ao } \\
\text { dia, junto com a refeição. }\end{array}$ \\
\hline $\begin{array}{l}\text { Fosfato de } \\
\text { cálcio }\end{array}$ & - 39\% (CAMPOS; PINHATI, 2013) & $\begin{array}{l}\text { O fosfato de cálcio também é livre de PMC, } \\
\text { seu preço é em torno de } 40,95 \text { reais, para } \\
60 \text { comprimidos. Ou seja, } 0,68 \text { centavos de } \\
\text { reais por dose. } \\
\text { Deve ser ingerido de } 1 \text { á } 2 \text { comprimidos du- } \\
\text { rante as refeições. }\end{array}$ \\
\hline
\end{tabular}

Fonte: autor (2019).

Referências: CAMPOS; PINHATI, 2013; ZENK; FRESTEDT; KUSKOWSKI, 2018. 


\section{Discussão}

O aumento da expectativa de vida, a diminuição da mortalidade e a redução da fecundidade resulta no envelhecimento da população e com isso aumento das taxas de doenças crônicas degenerativas, como a osteoporose.

No início do século XIX, a osteoporose significava "ossos fracos" ou "menos osso". A palavra osteoporose surgiu em 1830 , por um patologista francês, que realizou um estudo histológico de um osso osteoporótico. A doença popularizou-se como um sinal radiológico, que significava rarefação óssea, em fraturas causadas por traumas de baixa energia (SOUZA, 2010). É assintomática, pois a perda de massa óssea surge gradualmente. Em uma fase avançada gera dor crônica diminuindo a qualidade de vida (RADOMINSKI et al., 2017).

Os primeiros sinais de osteoporose pode ser uma fratura inexplicável durante as tarefas do dia-a-dia, considerando que estas mesmas tarefas não implicariam em fratura em um indivíduo saudável (SOUZA, 2010).

Também são sinais as dores, principalmente na coluna vertebral, aparecimento da curva dorsal, fraturas no rádio, vértebras e fémur. Em mulheres na menopausa, muitas vezes após a primeira fratura não é diagnosticada a osteoporose, aumentando a probabilidade de ocorrerem múltiplas fraturas (LOPES; CANHÃO; FONSECA, 2007).

A OMS estabeleceu um consenso sobre os grupos etários de risco e técnicas diagnósticas de osteoporose. O diagnós- tico e os riscos de fraturas devem ser analisados pelo T-score, que corresponde à diferença entre a DMO média de jovens normais e a DMO do indivíduo examinado, dividido pelo desvio-padrão da média de jovens normais. $O$ grau de diminuição de massa óssea é determinado através dos valores da DMO, que avalia a quantidade de mineral existente numa determinada área de osso (WORLD HEALTH ORGANIZATION, 1994)

O exame clínico padrão de análise densitométrica do fémur, envolve a análise de 4 regiões de interesse (ROI), com significado clínico, o triângulo de Ward, o trocânter, o colo do fémur e a região intertrocantérica. AROI do colo do fémur é região mais reprodutível e com maior padronização entre as marcas que comercializam aparelhos de densitometria, entretanto é a região femoral com maior incidência de fraturas osteoporóticas (63\%) (SANTOS, 2007).

\section{Tratamento}

O citrato de cálcio, também chamado de "sal azedo", é um sal originado do ácido cítrico $\mathrm{Ca}_{3}\left(\mathrm{C}_{6} \mathrm{H}_{5} \mathrm{O}_{7}\right)_{2}$, com peso molecular de $324,41 \mathrm{~g} / \mathrm{mol}$. O citrato de cálcio diminuiu os marcadores de reabsorção óssea (cross-link de colágeno, N-telopeptídeo (-30\%), C-telopeptídeo (-31\%), deoxipiridinolina livre (19\%) e N-telopeptídeo sérico (-8\%)), em comparação com o carbonato de cálcio em mulheres na pós-menopausa (KENNY et al., 2004).

Muitas vezes associa-se ácido cítrico, cálcio e ácido málico, para aumentar a biodisponibilidade e minimizar os efeitos 
adversos no trato gastrointestinal como vômito, irritação gástrica, obstipação, diarreia e refluxo (KENNY et al., 2004).

Para pacientes que usam antiácidos e tem nefrolitíase indica-se usar o citrato de cálcio, visto que é mais absorvível e acidificante da urina (SOUZA, 2010).

O carbonato de cálcio solubiliza em pH ácido, por isso o sal é pouco absorvido em pacientes que usam antiácidos e sua absorção ocorre nos segmentos proximais do intestino delgado. Apenas um terço é secretado pelo trato gastrointestinal, na saliva, bile e suco pancreático. $O$ cálcio endógeno e o cálcio não absorvido são excretados pelas fezes (KARP; KETOLA; LAMBERG-ALLARDT, 2009). Como efeito colateral se observa a constipação (RADOMINSKI et al., 2017).

Em relação ao cálcio de ostras $(40 \%$ de cálcio elementar), as conchas de moluscos bivalves da família Ostreidae, cuja sinonímia científica é Crassostrea risiphora, apresentam um elevado percentual de carbonato de cálcio, e, seu consumo favorece um desenvolvimento ambiental sustentável (AGRIPINO, 2016). Entretanto não pode ser usado por pacientes que usam antiácidos ou tem problemas renais, assim como o carbonato de cálcio.

As características físicas, químicas e térmicas do cálcio da concha de ostras foram determinadas. As cascas de mariscos possuem uma temperatura inicial de degradação superior ao carbonato de cálcio comercial e semelhança em perda de massa sendo que $95 \%$ da composição química das conchas é carbonato de cálcio, ou seja, este composto tem uma qualidade satisfatória e pode contribuir para um acesso maior da população ao cálcio oriunda da biodiversidade litorânea, diminuir agressão ambiental, gerando fonte de renda aos maricultores (HAMESTER, 2010).

O cálcio de ostra é solúvel eé absorvido em $\mathrm{pH}$ ácido e, em pacientes que utilizam antiácidos, o cálcio de ostras é pouco absorvido (CAMPOS; PINHATI, 2013).

Quanto ao fosfato de cálcio, os ossos são formados por moléculas de colágeno ligadas em cadeias lineares arranjadas em fibras. Entre estas moléculas há pequenos compartimentos intersticiais regularmente espaçados, onde estão presentes nanocristais de um sólido inorgânico chamado de fosfato de cálcio, que representa $65 \%$ da massa total do osso (GOMES et al., 2012). Ou seja, este sal juntamente com o cálcio, mantêm a integridade estrutural do esqueleto (SILVA; TEIXEIRA; GOLDBERG, 2004).

O fosfato de cálcio é utilizado em implantes dentários, osteoporose, substituição total de articulação, reconstrução crânio maxilo-facial e cirurgia de coluna (PAJAMÄKI, et al., 2003).

$\mathrm{O}$ sal fosfato de cálcio $\mathrm{Ca}_{10}\left(\mathrm{PO}_{4}\right)_{6}(\mathrm{OH})_{2}$ forma cristais de hidroxiapatita, de reabsorção muito lenta e remodelação óssea, com isso faz com que se mantenha no organismo durante anos. As biocerâmicas utilizadas para prótese de articulação, são feitas de $40 \%$ de hidroxiapatite e $60 \%$ de fosfato tricálcico (TCP), favorecendo a velocidade e qualidade da osseointegração, sua reabsorção, e mantendo a resistência a compressão (LOPES et al., 1999). 
O fosfato de cálcio é indicado para idosos com baixa ingestão de fósforo (casos raros), institucionalizados e com dificuldade de se alimentar (SOUZA, 2010).

Não é comum usar o gluconato de cálcio no tratamento de osteoporose e sim é usado no tratamento (via parenteral) de hipocalcemia com sintomas e sinais evidentes de aumento de neuroexcitabilidade (PAULA, 2003).

O magnésio é importante na fixação de cálcio nos ossos. A deficiência de magnésio pode causar ou agravar quadros de osteopenia e osteoporose no adulto e dificultar a calcificação correta dos ossos na infância e adolescência (SILVA, 2004). O magnésio é fundamental para o crescimento e desenvolvimento ósseo, bem como para a formação eficaz da trabécula, proporcionando resistência aos ossos. Entretanto ainda não existe dados conclusivos a respeito do magnésio na osteoporose. Sabe-se que o mesmo apresenta efeitos sobre a DMO devido à influência direta na atividade dos osteoblastos e osteoclastos, modulação do metabolismo ósseo, redução da liberação de citocinas inflamatórias e, auxílio na manutenção do equilíbrio ácido-básico do organismo (PAJAMÄKI et al., 2003).

O magnésio faz parte da estrutura óssea mineral juntamente com o cálcio e o fosfato e atua nos processos em que acontece a troca desses minerais entre o osso e os tecidos (ROSANOFF; DAI; SHAPSES, 2016). Além dos efeitos diretos sobre a estrutura e as células do esqueleto, uma deficiência de magnésio pode apresentar impactos indiretos sobre o osso, por afetar a homeostase de regu- ladores do cálcio e levar à hipocalcemia (PIRONI et al., 2009).

A hipomagnesemia geralmente se apresenta com hipocalcemia e a ingestão de cálcio afeta a retenção de magnésio e vice-versa. $\mathrm{O}$ magnésio impede a entrada de cálcio na célula quando o cálcio está presente em excesso bem como, magnésio impede a saída de cálcio durante condições deficientes em cálcio (ROSANOFF; DAI; SHAPSES, 2016). Deficiência de magnésio agrava ou leva à osteoporose. A redução do $\mathrm{pH}$ na hipomagnesemia pode alterar a bomba H-K-ATPase, provocando uma maior instabilidade óssea. Também, pode estar relacionada à diminuição na síntese de PTH, a baixos níveis sanguíneos de calcitriol e $1,25(\mathrm{OH})_{2} \mathrm{D}_{3}$ e a resistência a vitamina $\mathrm{D}$, que leva à osteoporose (GALLAGHER, 2001).

A deficiência de magnésio pode afetar a qualidade óssea pela diminuição da formação óssea, impedindo a formação de cristais ótimos e tendo um efeito negativo sobre o PTH (RUDE et al., 2005).

$O$ baixo teor de magnésio na dieta pode ser um fator de risco para osteoporose. Uma restrição dietética moderada de magnésio resultou em perda óssea. A deficiência profunda de magnésio, reduz $27 \%$ do conteúdo de magnésio ósseo e diminui as concentrações séricas de PTH (RUDE et al., 2005).

Os bisfosfonatos são análogos estruturais ao pirofosfato e atuam inibindo a reabsorção óssea, por meio da adsorção do fármaco à matriz óssea, ou seja, ligam-se à hidroxiapatita na superfície óssea e diminuem o número e a atividade 
dos osteoclastos. O acúmulo de bisfosfonatos no osso cria um reservatório, que leva a uma liberação continuada a partir do osso por meses ou anos após a suspensão do tratamento (LICATA, 1997).

O primeiro bisfosfonato largamente utilizado foi o alendronato. $\mathrm{O}$ mesmo reduziu significativamente a incidência de fraturas clínicas em pacientes com história de fraturas vertebrais. No entanto, uma pesquisa realizada em mais de 4.000 indivíduos com baixa DMO, sem história prévia de fraturas vertebrais, não foi capaz de demonstrar o mesmo benefício, exceto em uma subpopulação de mulheres com baixa DMO do fêmur (LIAO, et al., 2018).

Os agentes antirreabsortivos, como os bisfosfonatos e denosumabe, são os medicamentos mais prescritos atualmente para o tratamento da osteoporose, agem aumentando constantemente a DMO. Esses agentes são largamente prescritos, porém a dosagem deve ser utilizada com cuidado, pois pode ter um risco, de osteonecrose mandibular. $\mathrm{E}$, além disso, apresentam um custo elevado, visto que o PMC para o alendronato de sódio $(70 \mathrm{mg}$ ) é cerca de 53,61 reais, para 4 comprimidos. Deve ser administrado 1 comprimido, por semana, sempre no mesmo dia e horário, em jejum.

O fluoreto é altamente reativo e está presente na água sob a forma de sais de sódio e potássio. Sua ação na osteoporose começou a ser estudada na década de 60 , onde demonstrou aumentar a retenção de cálcio. $O$ fluoreto tem propriedades de estimular osteoblastos, tanto em número quanto em capacidade de formação de matriz óssea. No entanto, a dose efetiva é muito próxima da dose tóxica e há controvérsias a respeito da qualidade da formação do osso, isso limita sua utilização e, há relatos de osteomalácia associada ao uso de fluoretos (DOURADOR, 1999)

Altas doses de fluoreto (em torno de $80 \mathrm{mg} /$ dia), mostraram que o aumento de DMO não reflete necessariamente em aumento na força do tecido ósseo e, os índices de fratura entre pacientes tratados com fluoreto não são diferentes do grupo controle, ou seja, o fluoreto de sódio em altas doses, não é eficaz no tratamento da osteoporose (RIGGS; KHOSLA; MELTON, 1998).

O fluoreto de sódio produz aumento substancial na DMO, porém interfere na mineralização do osso, o que indica que são necessários mais estudos para definir a dose ideal para alcançar o efeito farmacológico desejado (REID et al., 2006).

$\mathrm{O}$ valor no mercado para o fluoreto de sódio (que contém $0,10 \mathrm{mg}$ de fluoreto de sódio + 0,0012 mg de cianocobalamina + 45mg de fosfato de cálcio tribásico para cada $1 \mathrm{~mL}$ da solução) é cerca de 15,34 reais, para um frasco de $100 \mathrm{~mL}$. Deve ser administrado $30 \mathrm{~mL}$ da solução, duas vezes ao dia antes das refeições (REID et al., 2006).

Para que ocorra a absorção intestinal de cálcio, a vitamina $\mathrm{D}$ é essencial. A vitamina $\mathrm{D}$ normalmente é deficiente ou insuficiente em idosos. Portanto a associação de ambos se torna uma estratégia simples e barata para prevenir fraturas osteoporóticas (WEBB; KLINE; HOLICK., 1988). 
O efeito benéfico da associação de cálcio e vitamina $\mathrm{D}$ foi comprovado em outra revisão (AVENELL et al., 2009). $A$ vitamina $D$ sozinha não mostrou efeito significativo de redução de fratura de quadril, fratura vertebral ou novas fraturas. Associada ao cálcio, apresentaram potencial em reduzir as fraturas de quadril, e fraturas não vertebrais e, não mostrou evidências benéficas nas fraturas vertebrais. A vitamina $D$ não aumentou os eventos adversos gastrointestinais. Portanto, idosos frágeis que vivem em instituições podem sofrer menos fraturas de quadril e outras fraturas não vertebrais se utilizarem vitamina $D$ com cálcio.

O tratamento de vitamina $\mathrm{D}$ e cálcio foi avaliado, para verificar se esta associação é justificável em termos de custo-efetividade em pacientes com idade superior a 60 anos que tenham osteoporose. Foi comparada a suplementação de cálcio e vitamina $\mathrm{D}$ com nenhum tratamento. Mulheres que faziam o tratamento aos 60 anos tinham um gasto anual de 9424 euros e as que não faziam tratamento, o custo com fraturas e/ou outras medicações, era de 9107 euros. Aos 70 anos esta média de valores se manteve (10553 euros para mulheres que faziam tratamento e 10427 euros para quem não fazia nenhum tratamento). Entretanto aos 80 anos as mulheres que faziam tratamento tinham um custo de 9242 euros e as que não faziam tratamento o custo foi de 9512 com fraturas osteoporóticas. Do ponto de vista econômico, o tratamento de vitamina $\mathrm{D}$ e cálcio deve ser utilizado em homens e mulheres que tenham osteoporose com idade superior a 60 anos, para diminuir o custo com fraturas osteoporóticas após os 80 anos (HILIGSMANN et al., 2015).

Recentemente em 2018 foi publicado uma meta-análise buscando comprovar os benefícios do uso de cálcio e vitamina D em 51.419 participantes. As doses variaram de 300 UI por dia a 100.000 UI a cada 1 a 4 meses para a vitamina $D$ e de 600 a $1.600 \mathrm{mg}$ por dia para o cálcio. Comparado com placebo, a suplementação com vitamina $\mathrm{D}$ por 3,5 a 5 anos diminuiu minimamente a incidência total de fratura, mas os achados foram imprecisos e não teve efeito estatisticamente significativo na fratura de quadril. $\mathrm{O}$ uso de vitamina $\mathrm{D}$ e cálcio por até 7 anos não foi significativo. Como efeitos adversos a vitamina $\mathrm{D}$ isolada ou com cálcio não teve efeito sobre a causa de outras doenças ou morte. $\mathrm{O}$ uso de cálcio sozinho por 2 a 4 anos não aumentou a incidência de cálculos renais, já a suplementação de cálcio e vitamina $\mathrm{D}$ por até 7 anos aumentou a incidência de cálculos renais. Portanto, em pacientes sem osteoporose ou deficiência de vitamina $\mathrm{D}$, não foi encontrado benefício com a suplementação de vitamina $D$ isolada ou com cálcio e a ingestão de apenas cálcio (KAHWATI et al., 2018).

\section{Conclusão}

O uso do cálcio apresenta muitos benefícios na prevenção e tratamento da osteoporose, especialmente ao ser associado ao magnésio e vitamina $\mathrm{D}$. Porém, cuidados devem ser tomados na 
indicação para pacientes com complicações em sua saúde. Visto que no varejo farmacêutico há diversos tipos de cálcios, do qual cada um tem peculiaridades diferentes, cabe aos prescritores a análise correta e minuciosa de cada paciente para definir a melhor indicação.

O carbonato de cálcio apresentou um efeito maior, em evitar fraturas vertebrais. $\mathrm{O}$ citrato de cálcio parece ser a melhor opção de compra para pacientes, prefeituras e instituições de saúde, pode ser usado por idosos que tem problemas renais e também aqueles que utilizam antiácidos. Também, tem uma absorção e biodisponibilidade superior ao carbonato em diversos dos estudos, sendo a escolha com o menor preço encontrado.

\section{Therapeutic management in calcium deficiency: a systematic review}

\section{Abstract}

Gradual bone loss causes osteopenia and its evolution leads to osteoporosis. Occurs decreased bone mass and deterioration of bone tissue microarchitecture, increased bone fragility and susceptibility to fractures. The purpose of this paper was to evaluate the differences between commercially available calcium presentations in their effectiveness, safety and cost. A systematic review was performed using the keywords: "calcium", "osteoporosis", "doses", "treatment", "randomized", "controlled" and "meta-analysis". The selection of publications was made based on inclusion and exclusion criteria. In total 379 relevant articles were identified. Of these, 10 met the selection criteria and were included in this study. Among the calcium salts are citrate, carbonate, fluoride, folinate, phosphate, and of the oysters, and bisphosphonates. Calcium associated with vitamin D and magnesium are also used. The use of calcium has many benefits in the prevention and treatment of osteoporosis, especially when associated with magnesium and vitamin D. Calcium citrate has been shown to be the best option as it can be used by elderly people with kidney problems and also by patients using antacids, showing higher absorption and bioavailability than carbonate, being the choice with the lowest price found.

Keywords: Calcium; Osteoporosis; Treatment; Pharmacoeconomics; Seniors.

\section{Referências}

AGRIPINO, A. A. Efeito do suplemento "Cálcio da Concha da ostra e fibra alimentar solúvel" na prevenção da osteoporose num modelo animal. 2016. 155 f. Tese (Doutorado) - Curso de Farmácia, Faculdade de Ciências e Tecnologia, Universidade Nova de Lisboa, Lisboa, Cap. 09, 2016.

AVENELL, A., et al. Vitamin D and vitamin $\mathrm{D}$ analogues for preventing fractures associated with involutional and post-menopausal osteoporosis. Cochrane Database of Systematic Reviews, v. 15, n. 2, CD000227, 2009. Doi: 10.1002/14651858.CD000227. pub2

BORISSOVA, A. M. European guidance for the diagnosis and management of osteoporosis in postmenopausal women. Springer, v. 24, n. 01, p. 23-57, jan. 2013.

CAETANO-LOPES, J; CANHÃO, H; FONSECA, J. E. Osteoblasts and bone formation. Acta Reumatológica Portuguesa, Lisboa, v. 32, p.103-110, abr. 2007. Disponível em: <https://www.ncbi.nlm.nih.gov/ pubmed/17572649>. Acesso em: 12 dez. 2019. 
CAMPOS, F. S; PINHATI, F. R. O Controle do Cálcio e a Hipocalcemia. Unifoa, Volta Redonda, v. 8, n. 23, p.77-85, 2013.

CARVAlHO, C. M. R. G; FONSECA, C. C. C; PEDROSA, J. I. Educação para a saúde em osteoporose com idosos de um programa universitário: repercussões. Cadernos de Saúde Pública, [s.l.], v. 20, n. 3, p.719-726, jun. 2004. Doi: 10.1590/S0102311X2004000300008.

COMPSTON, J.; et al. Osteoporosis in the European Community: Action Plan A report of the key next steps towards a Europe free from fragility fractures. 2003. Disponível em:<https://ec.europa.eu/health/ archive/ph_projects/2002/promotion/fp_promotion_2002_a1_04_en.pdf >. Acesso em: 19 dez. 2019.

DOURADOR, E. B. Osteoporose senil. Arquivos Brasileiros de Endocrinologia \& Metabologia, [s.l.], v. 43, n. 6, p.446-451, dez. 1999.

GALI, J. C. Osteoporose. Acta Ortopédica Brasileira, [s.l.], v. 9, n. 2, p.53-62, jun. 2001.

GALLAGHER, L. P. The potential for adverse drug reactions in elderly patients. Applied Nursing Research, v. 14, n. 4, p. 220-224, 2001. Doi: 10.1053/apnr.2001.26788

GOMES, L. C., et al. Síntese e caracterização de fosfatos de cálcio a partir da casca de ovo de galinha. Cerâmica, [s.1.], v. 58, n. 348, p.448-452, dez. 2012.

GRACITELLI, M. E. C.; et al. Paratormônio e osteoporose: encontrando o fio da meada. Bases fissiológicas para utilização do PTH no tratamento da osteoporose. Arquivos Brasileiros de Endocrinologia \& Metabologia, [s.l.], v. 46, n. 3, p.215-220, jun. 2002. Doi: 10.1590/S0004-27302002000300003

HAMESTER, D. Obtenção de carbonato de cálcio a partir de conchas de mariscos. Congresso brasileiro de engenharia e ciência dos materiais, 19, Campos do Jordão. São Paulo: Sociesc, p. 269, 2010.
HARVEY, J. A.; ZOBITZ, M. M.; PAK, C. Y. C. Dose dependency of calcium absorption: A comparison of calcium carbonate and calcium citrate. Journal of Bone and Mineral Research, [s.l.], v. 3, n. 3, p.253-258, 3 dez. 1988. Doi: 10.1002/jbmr.5650030303.

HEANEY, R. P.; et al. Absorbability and Cost Effectiveness in Calcium Supplementation. Journal of The American College of Nutrition, [s.1.], v. 20, n. 3, p.239-246, jun. 2001. Doi: 10.1080/07315724.2001.10719038.

HELLER, H. J., et al. Pharmacokinetics of Calcium Absorption from Two Commercial Calcium Supplements. The Journal of Clinical Pharmacology, v. 39, n. 11, p. 1151-1154, 1999.

HELLER, H. J., et al. et al. Pharmacokinetic and Pharmacodynamic Comparison of Two Calcium Supplements in Postmenopausal Women. Journal of Clinical Pharmacology. Dallas, v. 40, n. 11, p. 1237-1244. nov. 2000.

HILIGSMANN, M.; et al. Cost-effectiveness of vitamin $\mathrm{D}$ and calcium supplementation in the treatment of elderly women and men with osteoporosis. European Journal of Public Health, v. 25, n. 1, p. 20-25, 2015. Doi: 10.1093/eurpub/cku119

KAHWATI, L. C.; et al. Vitamin D, Calcium, or Combined Supplementation for the Primary Prevention of Fractures in CommunityDwelling Adults. Jama, [s.l.], v. 17, n. 319 (15), p.1600-1612, 17 abr. 2018. Doi: 10.1001/ jama.2017.21640.

KANIS, J. Diagnosis of osteoporosis and assessment of fracture risk. Lancet, v. 359, n. 9321, p.1929-1936, jun. 2002. Doi: 10.1016/ S0140-6736(02)08761-5.

KANIS, J. A.; et al. European guidance for the diagnosis and management of osteoporosis in postmenopausal women. Osteoporosis International, v. 19, n. 7, p. 1103-1104, 2008.

KARP, H. J.; KETOLA, M. E.; LAMBERGALLARDT, C. J. E. Acute effects of calcium 
carbonate, calcium citrate and potassium citrate on markers of calcium and bone metabolism in young women. British Journal of Nutrition, v. 102, n. 9, p.1341-1347, 19 jun. 2009. Doi: 10.1017/S0007114509990195

KENNY, A. M. et al. Comparison of the effects of calcium loading with calcium citrate or calcium carbonate on bone turnover in postmenopausal women. Osteoporosis International, v. 15, n. 4, p.290-294, 1 abr. 2004. Doi: 10.1007/s00198-003-1567-0.

KRÖGER, H; KÄRKKÄINEN, M; HONKANEN, R. Calcium and Vitamin D in Promotion of Postmenopausal Bone Health. Women's Health, v. 6, n. 6, p.773-776, nov. 2010. DOI: $10.2217 /$ whe.10.51

LIAO, E. Y. et al. Clinical characteristics associated with bone mineral density improvement after 1-year alendronate/vitamin d3 or calcitriol treatment: Exploratory results from a phase 3 , randomized, controlled trial on postmenopausal osteoporotic women in China. Medicine, v. 97, n. 31, p. e11694, 2018. Doi: 10.1097/MD.0000000000011694.

LICATA, A. A. Bisphosphonate therapy. American Journal of the Medical Sciences, v. 313, n. 1, p. 17-22, 1997.

LOPES, M. A. et al. Osteocompatibility and In Vivo Evaluation of Glass Reinforced Hydroxyapatite Composite. Bioceramics, p. 421-424, out. 1999. Doi: 10.1142/9789814291064_0101

PAJAMÄKI, J. K; LINDHOLM, T. S; ANDERSSON, Ö. H; KARLSSON, K; VEDEL, E; PAULA, F. J. A; FOSS, M. C. Tratamento da hipercalcemia e hipocalcemia. Medicina (Ribeirao Preto Online), v. 36, p. 370-374, 2003.

PIRONI, L. et al. The complex relationship between magnesium and serum parathyroid hormone: A study in patients with Chronic Intestinal Failure. Magnesium Research, v. 22 , n. 1, p. 37-43, 2009. Doi: 10.1684/ mrh.2009.0158
RADOMINSKI, S. C, et al. Diretrizes brasileiras para o diagnóstico e tratamento da osteoporose em mulheres na pós $\square$ menopausa. Revista Brasileira de Reumatologia, v. 57, p.452-466, 2017. Doi: 10.1016/j. rbr.2017.06.001.

REID, I. R, et al. Randomized Controlled Trial of Calcium in Healthy Older Women. The American Journal of Medicine, v. 119, n. 9, p. 777-785, 2006. Doi: 10.1016/j. amjmed.2006.02.038.

RIGGS, B. L.; KHOSLA, S; MELTON, L. J. A unitary model for involutional osteoporosis: Estrogen deficiency causes both type I and type II osteoporosis in postmenopausal women and contributes to bone loss in aging men. Journal of Bone and Mineral Research, v. 13 , n. 5, p. 763-773, 1998. Doi: 10.1359/jbmr.1998.13.5.763.

ROSANOFF, A; DAI, Qi; SHAPSES, S. A. Essential Nutrient Interactions: Does Low or Suboptimal Magnesium Status Interact with Vitamin D and/or Calcium Status? Advances In Nutrition, v. 7, n. 1, p.25-43, 1 jan. 2016. Doi: 10.3945/an.115.008631.

RUDE, R. K. et al. Dietary magnesium reduction to $25 \%$ of nutrient requirement disrupts bone and mineral metabolism in the rat. Bone, v. 37, n. 2, p. 211-219, 2005. Doi: 10.1016/j.bone.2005.04.005.

SANTOS, L. M. F. Análise comparativa dos parâmetros quantitativos do osso osteoporótico - modelo computacional de remodelação óssea vs resultados clínicos de dexa. 2007. 111 f. Dissertação (Mestrado) - Curso de Engenharia Biomédica, Faculdade de Medicina, Universidade de Lisboa, Lisboa, Cap. 8, 2007.

SILVA, C. C.; TEIXEIRA, A. S; GOLDBERG, T. B. L. Impacto da ingestão de cálcio sobre a mineralização óssea em adolescentes. Revista de Nutrição, v. 17, n. 3, p.351-359, set. 2004. Doi: 10.1590/S141552732004000300008 . 
SOUZA, M. P. G. Diagnóstico e tratamento da osteoporose. Revista Brasileira de Ortopedia, v. 45, n. 3, p.220-229, jun. 2010.

WEBB, A. R.; KLINE, L.; HOLICK, M. F. Influence of Season and Latitude on the $\mathrm{Cu}-$ taneous Synthesis of Vitamin D 3: Exposure to Winter Sunlight in Boston and Edmonton Will Not Promote Vitamin D 3 Synthesis in Human Skin. The Journal of Clinical Endocrinology and Metabolism, v. 67, n. 2, p. 373-378, 1988. Doi: 10.1210/jcem-67-2-373.

WORLD HEALTH ORGANIZATION, 1994, Geneva. Assessment of fracture risk and its application to screening for postmenopausal osteoporosis: report of a WHO study group. Roma: Who Technical Report Series, p. 129, 1994.

ZENK, J. L; FRESTEDT, J. L.; KUSKOWSKI, M. A. Effect of Calcium Derived from Lithothamnion sp. on Markers of Calcium Metabolism in Premenopausal Women. Journal of Medicinal Food, v. 21, n. 2, p.154-158, fev. 2018. Doi: 10.1089/jmf.2017.0023.

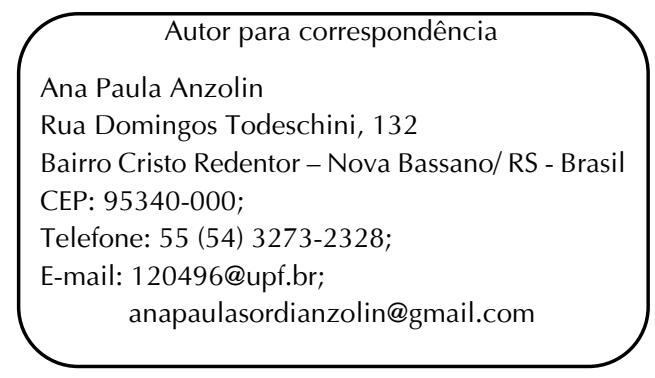

Financiamento: recursos próprios. 